\title{
2773. Research and application of Volterra series theory in rolling bearing fault state feature extraction
}

\author{
Haitao Wang', Zhenya Kang ${ }^{2}$, Lichen $\mathrm{Shi}^{3}$, Kun Wang ${ }^{4}$, Xiao Zhang \\ Xi' an University of Architecture and Technology, Electrical and Mechanical College, \\ Shaanxi Xi'an, 710055, China \\ ${ }^{3}$ Corresponding author

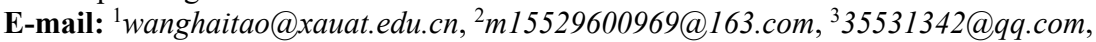 \\ 4546453921@qq.com, 5410183636@qq.com \\ Received 18 April 2017; received in revised form 25 November 2017; accepted 4 December 2017
} DOI https://doi.org/10.21595/jve.2017.18504

Check for updates

\begin{abstract}
Due to the generally strong non-linear characteristics of bearing failure, leading to overall mechanical system failure, fault state feature extraction is difficult. In this paper, a fault feature extraction method based on the Volterra series kernel under multi-pulse excitation is proposed. To avoid reliance on simplified models based on traditional mechanics, a nonlinear Volterra series model was constructed by introducing the input and output signals of the system, and using a low-order Volterra series kernel from the time domain and frequency domain, which was then solved using a multi-pulse excitation method. Furthermore, the state of the rolling bearing was determined using different characteristics of the corresponding generalized frequency response, and the current fault stage was inferred. The rolling bearing failure was validated experimentally, and it was shown that the Volterra series model can be more easily used to extract fault characteristics and trends of a rolling bearing in comparison to the traditional wavelet algorithm, therefore serving as a better method for fault prediction.
\end{abstract}

Keywords: Volterra series kernel, multiple-pulse excitation method, rolling bearings, fault diagnosis.

\section{Introduction}

Rolling-element bearings, also called rolling bearings, are the core components of rotating machinery. When failure of a rolling bearing occurs, the vibrational signal is usually strongly non-linear with non-stationary features $[1,2]$.

Therefore, it is possible to extract the fault characteristics from this type of signal in order to accurately differentiate fault types and failure development trends. Traditional feature extraction methods for nonlinear non-stationary signals have included the wavelet transform (WT) [3-5], singular value decomposition (SVD) [6], empirical mode decomposition (EMD) [7, 8], and the Hilbert-Huang transform [9]. While these methods have been widely used, they do not provide complete extraction of the fault information and exhibit or adaptive filtering. Moreover, nonconformity of the fault frequency drift can occur due to the existence of bias in the simplified mechanical model established using the theoretical failure values and actual system outputs.

A number of time-frequency manifold (TFM) techniques have been proposed [10] including the use of cross features from nonlinear data [11], chaotic feature space [12], ICD and adjustable Q-factor wavelet transforms [13], dominant trend based logistic regression (DTLR), transient modeling, and the new non-linear time-frequency feature extraction method using the Levenberg-Marquardt (LM) method for parameter identification [14]. However, most of the above methods have only been optimized for feature extraction from the vibrational data obtained under certain conditions and using specialized equipment. Optimization of a number of factors have not previously been considered: 1) theoretical mechanics to simplify the model and real system error; 2) the fault characteristic signal of the nonlinear non-stationary case; and 3) the fault trend evolution.

Based on the fault feature extraction method of the Volterra series kernel model and the multi-pulse excitation method, a different method of fault information feature extraction is 
proposed using a nonlinear transfer function $[15,16]$. The output signal can be used to establish a non-linear closed-loop system [17] to accurately describe the transmission characteristics of a nonlinear bearing system. In the fault diagnosis model [18-21], the Volterra time domain kernel (GIRF) and frequency domain kernel (GFRF) can be used to determine the dynamic characteristics of a nonlinear system. The problem of intuitionistic representation of the time and frequency domain kernel function was solved using the bispectrum method [22-24] and the validity of the method was verified experimentally.

\section{The multiple excitation algorithm}

The Volterra series can be theoretically determined using numerical algebra and infinite length algebraic sum of the multidimensional convolution. The specific formula is defined as:

$y(t)=h_{0}(t)+\sum_{k=1}^{\infty} \int_{0}^{\infty} \ldots \int_{0}^{\infty} h_{k}\left(\tau_{1}, \tau_{2}, \ldots, \tau_{k}\right) \prod_{i=1}^{k} \mu\left(t-\tau_{i}\right) d \tau_{i}$,

where $h_{k}\left(\tau_{1}, \tau_{2}, \ldots, \tau_{k}\right)$ is the core of the $k$-th order Volterra series.

From the above equation, the kernel is the key to solving the series development, and thus the foundation of the series element. For a discrete nonlinear time invariant system, the series can be represented as:

$y(n)=y_{0}+\sum_{n=1}^{\infty} \sum_{i_{1}=0}^{\infty} \ldots \sum_{i_{k}=0}^{\infty} h_{k}\left(i_{1}, \ldots, i_{k}\right) \prod_{k=1}^{\infty} \mu\left(n-i_{k}\right)$.

In order to ensure operability, system modeling should be carried out within certain value limits. The effects are not calculated based on the dc component such that Eq. (2) can be rewritten as:

$y(n)=y_{0}+\sum_{n=1}^{N} \sum_{m_{1}=0}^{M-1} \ldots \sum_{m_{n}=0}^{M-1} h_{0}\left(m_{1}, \ldots, m_{k}\right) \prod_{k=1}^{\infty} \mu\left(n-m_{k}\right)+e(k)$,

where $N$ is the highest order of the non-linear system, $M$ is the length of memory of the impulse response, and $e(k)$ is the truncation error. Assuming the system is nonlinear, Eq. (3) can be used and the first unit of a certain gain pulse will be the system input, namely, $\mu_{0}(t)=a_{\delta} \delta(t)$ the following formulae can then be used to determine the first-order kernel of the system:

$\mu_{0}(t)=a_{\delta} \delta(t) \rightarrow y_{0}(t)$,

$y_{0}(t)=a_{\delta} h_{1}(t)$,

where $a_{\delta}$ is the pulse stimulated amplitude.

From the equation:

$h_{1}(\tau)=\frac{y_{0}(t)}{a_{\delta}}$

There are both $h_{1}\left(\tau_{1}\right)$ and $h_{2}\left(\tau_{1}, \tau_{2}\right)$ assuming of the Volterra series of the kernel, with two different incentives as input to solve and discuss. The final formula is given as follows:

$\mu_{0}(t)=a_{\delta} \delta(t) \rightarrow y_{0}(t)$,

$y_{0}(t)=a_{\delta} h_{1}\left(\tau_{1}\right)+a_{\delta}^{2} h_{2}\left(\tau_{1}, \tau_{2}\right)$, 
$\mu_{1}(t)=2 a_{\delta} \delta(t) \rightarrow y_{1}(t)$,

$y_{1}(t)=2 a_{\delta} h_{1}\left(\tau_{1}\right)+4 a_{\delta}^{2} h_{2}\left(\tau_{1}, \tau_{2}\right)$.

Expressed in matrix form and substituting Eqs. (7)-(10) into the following formula:

$\left[\begin{array}{cc}a_{\delta} & a_{\delta}^{2} \\ 2 a_{\delta} & 4 a_{\delta}^{2}\end{array}\right]\left[\begin{array}{c}h_{1}\left(\tau_{1}\right) \\ h_{2}\left(\tau_{1}, \tau_{2}\right)\end{array}\right]=\left[\begin{array}{l}y_{0}(t) \\ y_{1}(t)\end{array}\right]$

where $h_{1}(\tau)$ can be written as:

$h_{1}\left(\tau_{1}\right)=\frac{4 y_{0}(t)-y_{1}(t)}{2 a_{\delta}}$.

In the same way, $h_{2}\left(\tau_{1}, \tau_{2}\right)$ can be represented by:

$h_{2}\left(\tau_{1}, \tau_{2}\right)=\frac{y_{1}(t)-2 y_{0}(t)}{2 a_{\delta}^{2}}$.

Given $h_{1}\left(\tau_{1}\right), h_{2}\left(\tau_{1}, \tau_{2}\right)$ and $h_{3}\left(\tau_{1}, \tau_{2}, \tau_{3}\right)$ and assuming the Volterra series kernel, the series can be written as:

$y(t)=h_{0}+\sum_{n_{1}=-\infty}^{n_{1}=+\infty} h_{1}\left(\tau_{1}\right) \cdot x\left(n-n_{1}\right)+\sum_{n_{1}=-\infty}^{n_{1}=+\infty} h_{1}\left(\tau_{1}, \tau_{2}\right) \cdot x\left(n-n_{1}\right) x\left(n-n_{2}\right)+\cdots$

where $h_{3}\left(\tau_{1}, \tau_{2}, \tau_{3}\right)$ is the third order time domain kernel of the non-linear system.

Using three different pulse excitations $h_{1}\left(\tau_{1}\right), h_{2}\left(\tau_{1}, \tau_{2}\right)$, and $h_{3}\left(\tau_{1}, \tau_{2}, \tau_{3}\right)$ as the input signals, the series can be solved using the following formula:

$\left[\begin{array}{ccc}a_{\delta} & a_{\delta}^{2} & a_{\delta}^{3} \\ 2 a_{\delta} & 4 a_{\delta}^{2} & 8 a_{\delta}^{3} \\ 3 a_{\delta} & 9 a_{\delta}^{2} & 27 a_{\delta}^{3}\end{array}\right]\left[\begin{array}{c}h_{1}\left(\tau_{1}\right) \\ h_{2}\left(\tau_{1}, \tau_{2}\right) \\ h_{3}\left(\tau_{1}, \tau_{2}, \tau_{3}\right)\end{array}\right]=\left[\begin{array}{l}y_{0}(t) \\ y_{1}(t) \\ y_{2}(t)\end{array}\right]$

Based on the Vandermonde matrix and using the numerical method to solve the Volterra first-order kernel, the formula can be represented as:

$h_{1}\left(\tau_{1}\right)=\frac{18 y_{0}(t)-9 y_{1}(t)+2 y_{2}(t)}{6 a_{\delta}}$.

In the same way, we get:

$h_{2}\left(\tau_{1}, \tau_{2}\right)=\frac{4 y_{1}(t)-5 y_{0}(t)-y_{2}(t)}{2 a_{\delta}^{2}}$,

$h_{3}\left(\tau_{1}, \tau_{2}, \tau_{3}\right)=\frac{3 y_{0}(t)+y_{2}(t)-3 y_{1}(t)}{6 a_{\delta}^{3}}$.

Different pulse excitation inputs into the system will result in different Volterra series outputs. If the system input is greater than the third-order pulse, i.e., an advanced kernel, then the model becomes computationally intensive and solving the inverse matrix $[A B]$ of the coefficient matrix $[A B]^{-1}$ falls into the curse of dimensionality. 


\section{Verification of the method}

For any continuous nonlinear time invariant system under initial conditions equal to zero, the input signal energy is limited to the system response that can be described by the Volterra series. It is assumed that there exists a linear single degree of freedom function, as shown by:

$c_{y} \frac{d y(t)}{d t}=f(y)+\mu(t)$

where $f(y)$ is always present and unique within the domain of definition.

When time $t=T$, the pulse excitation is the input function of the system $u(t)$ and the input function can be described by the following:

$\mu(t)=a_{\delta} \delta(t-T)$

where $a_{\delta}$ is the pulsed excitation amplitude.

When the system reaches $t=T$ at amplitude $a_{\delta}$ after the stimulation, the system will produce a finite amplitude jump in response to:

$y\left(T^{+}\right)=y\left(T^{-}\right)+\frac{a_{\delta}}{c_{y}}$

where $y\left(T^{+}\right)$represents the system response after excitation $T$ and $y\left(T^{-}\right)$is the system response before excitation $T$.

Integrating from $t=T^{-}$to $t=T^{+}$, it is possible to simulate the response of the system at the approximate time $t=T$. Eq. (21) can then be converted to:

$c_{y} \frac{d y(t)}{d t}=f(y)+c_{u} \mu(t)$

where $\mu(t)=a_{\delta} \delta(t-T)$ will produce a discontinuous system response, given by:

$y\left(T^{+}\right)=y\left(T^{-}\right)+\frac{c_{u} a_{\delta}}{c_{y}}$.

According to the above equation, the input function can be expressed as a piecewise function according to the time of the pulse excitation function applied to the system:

$$
\begin{cases}a_{\delta}\left[\frac{1}{\omega_{\delta}}-\frac{3\left(t-t_{\delta}\right)^{2}}{\omega_{\delta}^{3}}-\frac{2\left(t-t_{\delta}\right)^{3}}{\omega_{\delta}^{4}}\right], & t_{\delta}-\omega_{\delta}<t \leq t_{\delta} \\ a_{\delta}\left[\frac{1}{\omega_{\delta}}-\frac{3\left(t-t_{\delta}\right)^{2}}{\omega_{\delta}^{3}}+\frac{2\left(t-t_{\delta}\right)^{3}}{\omega_{\delta}^{4}}\right], & t_{\delta} \leq t \leq t_{\delta}+\omega_{\delta} \\ 0, & \text { others. }\end{cases}
$$

Eq. (24) can be approximated to simulate the system impulse input excitation function $\mu(t)=a_{\delta} \delta(t-T)$ at $t=t_{\delta}$ and width $2 \omega_{\delta}$. Integrating Eq. (24), it can be found that the maximum value of the impulse excitation $\mu(t)$ appears at $t=t_{\delta}$, and the derivative at the boundary is zero at $t_{\delta} \pm \omega_{\delta}$.

Based on the above analysis, when the system exhibits a pulse function, the response status approaches the ideal unit pulse. By applying multiple pulse excitation to the system, the theoretical change in response of the system state is also close to the ideal unit impulse. Therefore, a solution based on the pulse input using the theoretical kernel method from theory to data calculation is 
feasible and $\mu(t)=a_{\delta} \delta(t-T)$.

\section{Wavelet comparison experiment}

To experimentally validate the model, a test bench (Case Western Reserve University) was used, as shown in Fig. 1. The setup included a $2 \mathrm{HP}$ motor $(1.5 \mathrm{~kW})$, torque sensor/decoder, power meter, and electronic controller (not shown in the figure). The bearing support was attached to the shaft of the motor, drive-end bearing for SKF6205, and fan end bearing for SKF6203, and the ball bearing damage point was detected at three different positions: $90^{\circ}, 0^{\circ}$, and $-90^{\circ}$. An acceleration sensor was placed above the fan end of the motor and drive end bearing and used to collect the fault vibration signal of the bearing. The vibration signal was recorded using a 16-channel recorder, and power and speed were both measured using a torque sensor. A normal bearing and ball bearing were chosen for the analysis, and data was obtained using a load of $1492 \mathrm{~W}$ and a speed of $1750 \mathrm{r} / \mathrm{min}$.

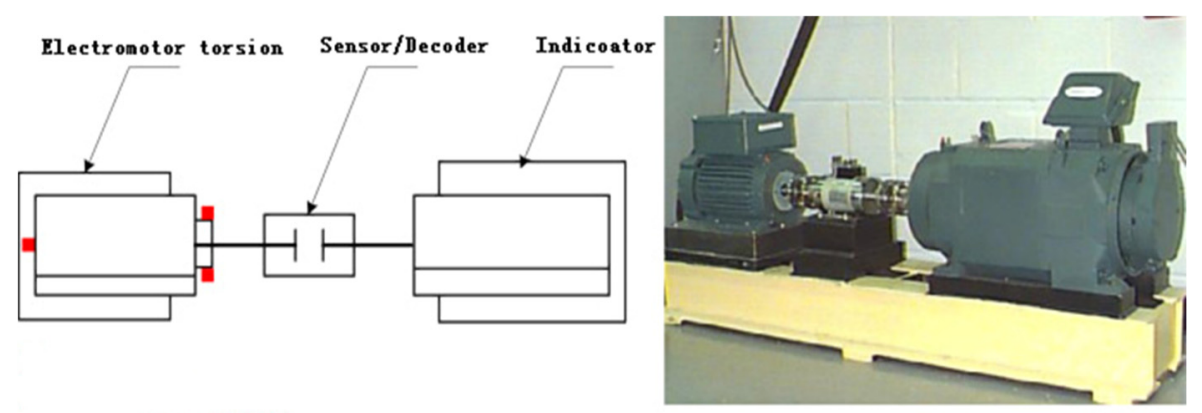

Fig. 1. Rolling bearing fault simulation test bench

A wavelet transform was adopted to diagnose the ball bearing signal. The wavelet base was used for the signal decomposition and to amplify the signal of the failure of the bearing in order to determine the failure frequency. Since the working frequency and bearing failure frequency are small, the low frequency range signal was amplified. The signal was then decomposed into four layers by the wavelet transform, and analyzed using Hilbert envelope spectral analysis.

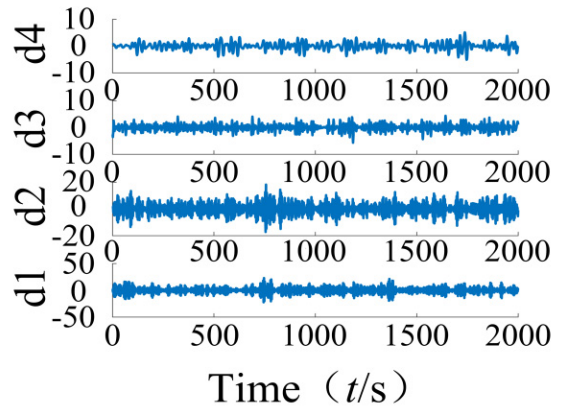

a) 4 layer wavelet decomposition

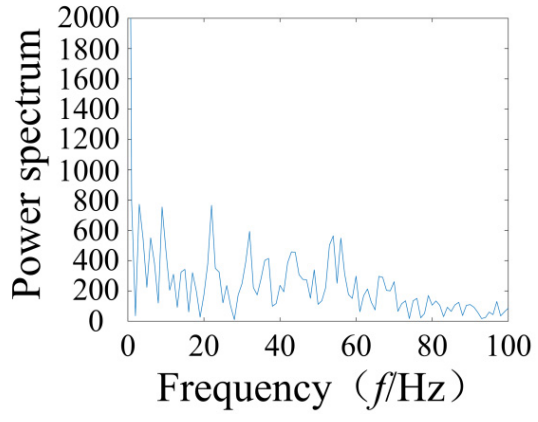

Spectrum

Fig. 2. Fault bearing wavelet decomposition and feature spectrum diagram

The wavelet decomposition was used to assess the bearing wear signal and produce the spectral diagram, as shown in Fig. 2. From observation, the effects of the frequency spectrum on the weak rolling bearing failure are poor, and clutter within the spectrum affects the overall results of the analysis such that fault feature extraction from the system is unclear. Moreover, when using the system signal based on the wavelet analysis, the corresponding wavelet base must be selected based on practical experience. This is significant since the selected wavelet base will strongly 
affect the system. Therefore, to obtain accurate results from the analysis, the correct wavelet base must be chosen, and this process of fault diagnosis can be tedious.

\section{Experimental demonstration of the proposed Volterra series method}

The Volterra series kernel can be assessed using the multi-pulse excitation method to avoid the disadvantages associated with the wavelet decomposition. Using the signal collected from Case Western Reserve University and based on the multi-pulse excitation method, the Volterra series kernel was used to investigate a normal bearing and ball bearing. Three conditions were used: a memory length of the first-order time-domain kernel of 8 and length of the kernel vector of 8; memory length of the second-order time-domain kernel of 5 and kernel vector length of 15; memory length of the second-order time-domain kernel of 5 and vector length of 35 .

The multi-pulse excitation method was used to analyze the rolling bearing signals, and it was assumed the series kernels $h_{1}, h_{2}$, and $h_{3}$ were present. First, the system inputs a pulse excitation function $\mu_{0}(t)=\delta(t)$, and the system output is $y_{0}$. Then, the two pulse excitations are applied to the system $u_{1}(t)=\delta(t)+\delta(t-T)$, where $T$ represents the pulse time delay, and the system generates another output $y_{1}(t)$. Similarly, three additional pulses $u_{2}(t)=\delta(t)+\delta(t-T)+\delta(t-2 T)$ with delayed impulses were applied to the system and again, the system generated the output $y_{2}(t)$.

The second-order time-domain kernel of the system can be obtained using Eq. (17). The multi-pulse excitation method was used to analyze the rolling bearing signals from the experiment, and a Matlab simulation was then used to obtain the second-order time-domain kernel characteristic curve as shown in Fig. 3. It can be observed that the second-order nuclear time domain spectra of the normal bearing and faulty bearing are clearly different as the numbers of sharp points on each spectrum are quite distinct.

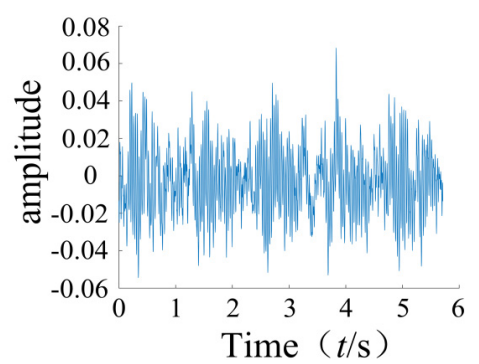

a) Normal bearing

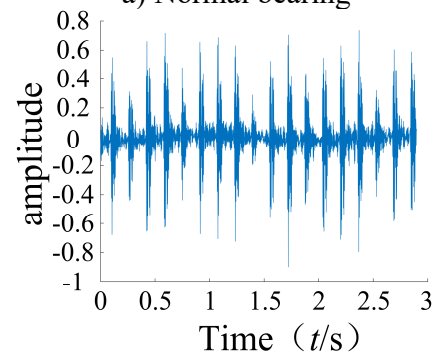

c) Outer ring failure

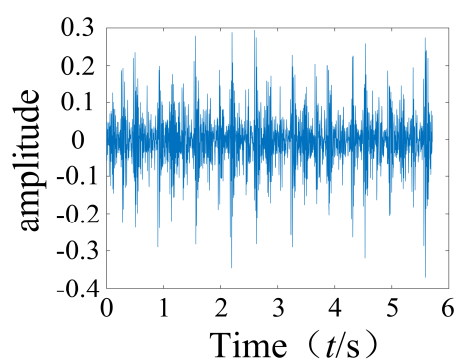

b) Inner ring failure

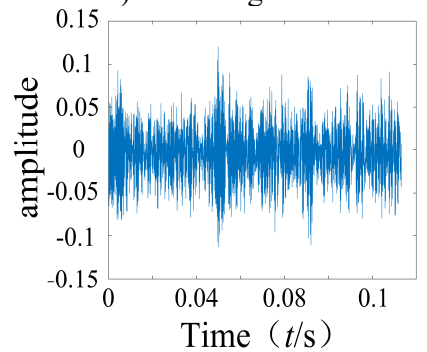

d) Roller failure

Fig. 3. Normal bearing and different periods during failure of the second order nuclear time-domain response

A comparison of the normal bearing and faulty bearing using the second-order time domain is presented in Fig. 4. It was found that the frequencies at which the highest peak appear are $0.3 \mathrm{~Hz}$, $0.8 \mathrm{~Hz}$, and $0.1 \mathrm{~Hz}$. The sites of failure can be clearly distinguished, and directly show huge differences in frequency between the highest peaks and different fault areas on the bearing. 


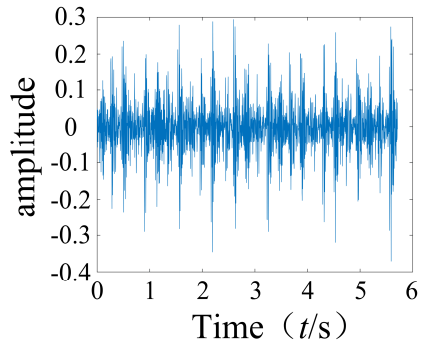

a) Inner ring failure

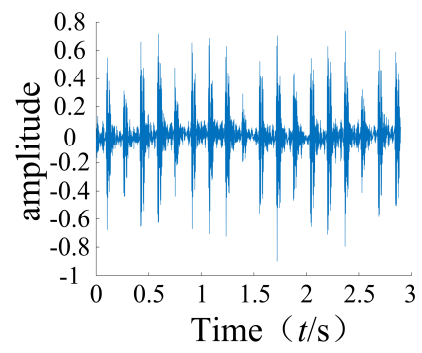

b) Outer ring failure

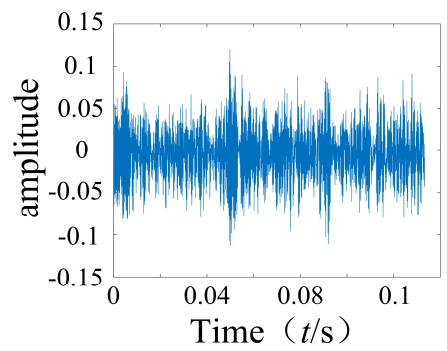

d) Roller failure

Fig. 4. Response of second order nuclear time domain in different parts

After several tests, it was found that the first-order time-domain kernels of the normal condition and the faulty condition were very similar for the time domain kernel (GIRF), and therefore difficult to distinguish. As such, they will not be discussed further in this paper. The graphs of the second-order kernel clearly show differences in the spikes on the graphs of the faulty bearing compared to the normal bearing (Figs. 3-4), and the number of spikes is greater for the faulty bearing. It was shown that non-linear faults could be detected using the low order kernel of the Volterra series.

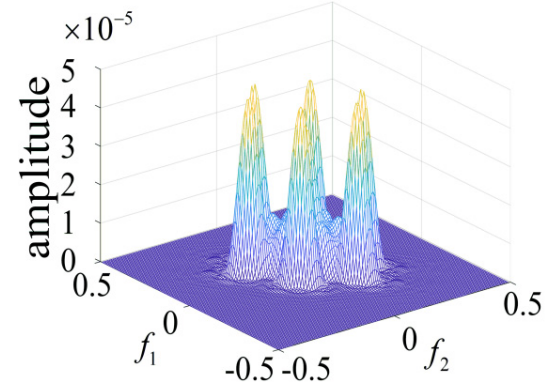

a) Normal bearing

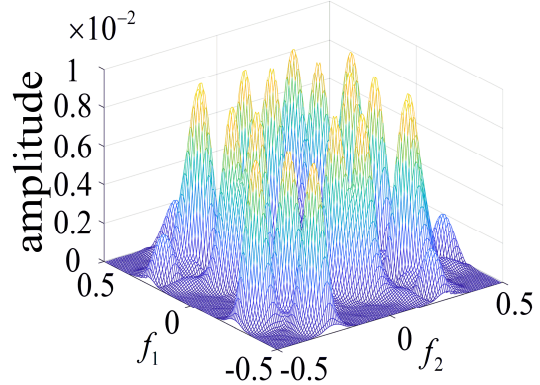

c) Outer ring failure

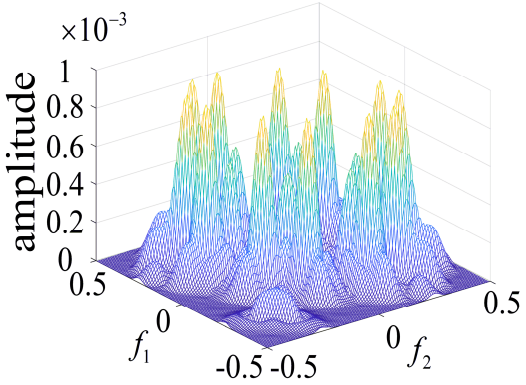

b) Inner ring failure

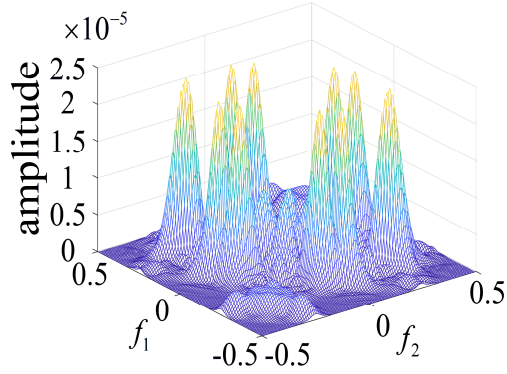

d) Roller failure

Fig. 5. Curves of the normal bearing and different parts of the faulty bearing based on the second order nuclear frequency domain response

In order to more intuitively observe the differences between bearing states, the bearing running states were observed. The second-order nuclear frequency response diagram (GFRF) and contour curves of the normal bearing and bearing inner ring failure, outer ring failure and ball bearing failure, are presented in Figs. 5-6. It can be observed that the second-order nuclear frequency domains of the normal and faulty bearings are clearly different. The bearing failure site is more clearly seen in the response contour curves. The frequency domain response diagram and second-order core contour figure effectively shows the position of the frequency secondary coupling, and can therefore confirm that the characteristics of the normal bearing and different parts of the faulty bearing system are fundamentally different. 


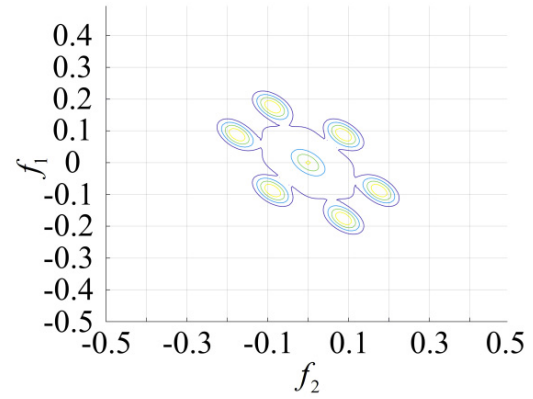

a) Normal bearing

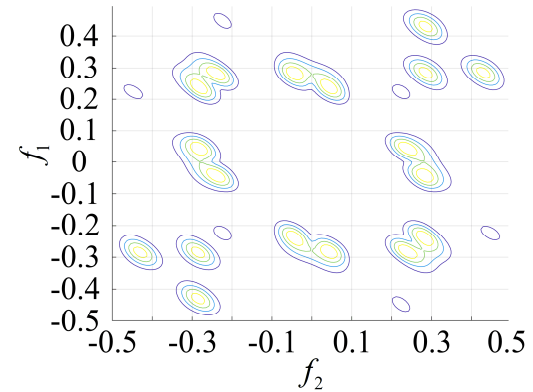

c) Outer ring failure

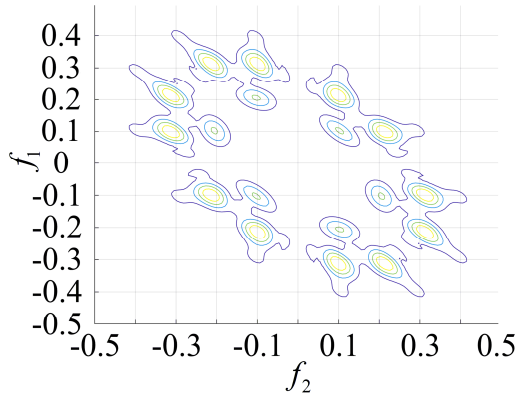

b) Inner ring failure

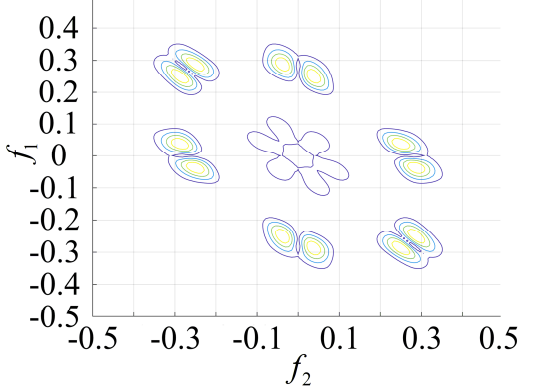

d) Roller failure

Fig. 6. Contours of the normal bearing and different parts of the faulty bearing based on the second-order amplitude-frequency domain response

A comparison of the normal bearing versus different parts of the faulty bearing, based on the second-order frequency domain, are shown in Fig. 5. It was found that the frequency points at which the highest peaks appear are at $3.5 \times 10^{5} \mathrm{~Hz}, 0.8 \times 10^{3} \mathrm{~Hz}, 0.8 \times 10^{2} \mathrm{~Hz}$ and $2.0 \times 10^{5} \mathrm{~Hz}$. This is consistent with theoretical calculations of the frequency of failure according to frequency and peak number, and can clearly distinguish between the normal and faulty bearings (Fig. 6). The second-order amplitude-frequency response profile of the normal bearing and different parts of the faulty bearing clearly show differences in the number of peaks and the dispersions of the spectra vary greatly. The second-order nuclear frequency domain diagram of the faulty bearing and its separate components depict the differences in system characteristics between the bearings.

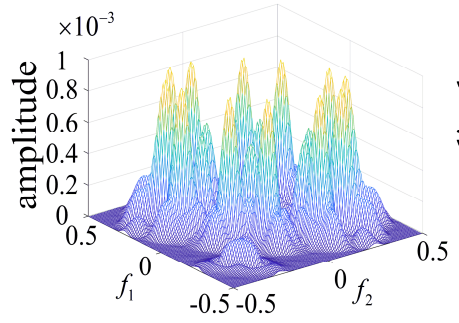

a) Inner ring failure

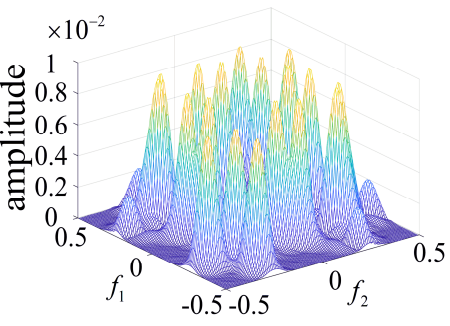

b) Outer ring failure

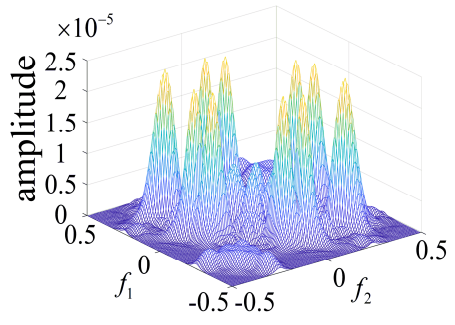

c) Roller failure

Fig. 7. Response of the second order nuclear frequency domain in different parts of the rolling bearing

The points at which the highest frequency peaks appear were found at $0.8 \times 10^{3} \mathrm{~Hz}, 0.8 \times 10^{2} \mathrm{~Hz}$ and $2.0 \times 10^{5} \mathrm{~Hz}$ (Fig. 7(a) and Fig. 5(b) and (c)). An intuitive description of the different parts of the fault bearing showing the peak frequency points suggest the difference is very clear. The degree of each coupling difference is presented in Fig. 8, which clearly shows the sites of failure.

Based on the Volterra series rolling bearing fault diagnosis, the multi-pulse excitation method can adequately represent the nonlinearity of the system. For different types of system, the method can be used to determine changes in the system using the second order Volterra time domain 
kernel and third order Volterra time domain kernel. Alternatively, the frequency domain kernel can be used under circumstances where the first order Volterra time domain kernel is difficult to determine, thereby providing richer fault information. It can also be used to determine the operating state of the system via changes in the Volterra nuclear frequency domain figure, obtained using the unique pulse excitation method (Fig. 7).

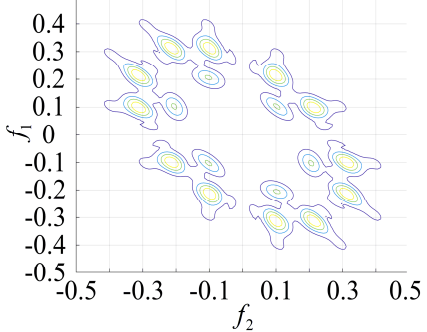

a) Inner ring failure

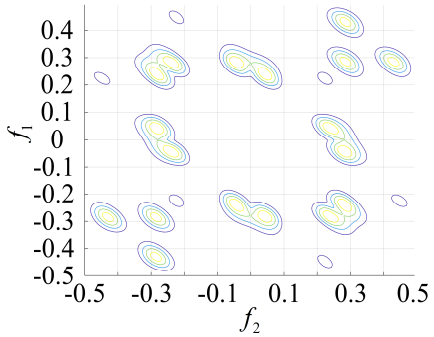

b) Outer ring failure

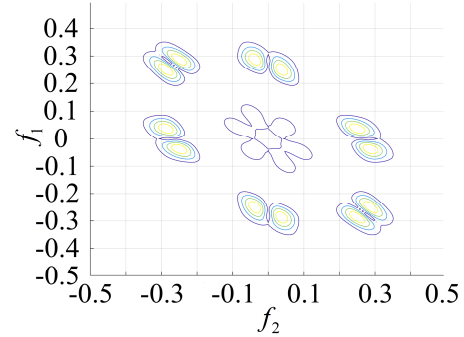

c) Roller failure

Fig. 8. Contours of the different faulty bearing parts for the second-order amplitude-frequency response

\section{Fault quantification and anti-noise analysis}

\subsection{Quantitative study on the fault evolution}

In this paper, the normal bearing and bearings with roller faults of various sizes, fault diameters of either $0.18 \mathrm{~mm}$ or $0.36 \mathrm{~mm}$ and a fault depth of $0.28 \mathrm{~mm}$, were used. The drive load was taken as 0 and the drive speed set to $1797 \mathrm{r} / \mathrm{min}$. The input signal to the system was either set to $\sigma(t)$ or $\sigma(t)+\sigma(t-1)$, and as the system was run, the vibration output signal was collected using an acceleration sensor. Experimental data were sampled, and 2000 sets of data were intercepted in the sample data for simulation analysis. The multi-pulse excitation method was then used to analyze the input and output data in MATLAB, and the characteristics of the normal bearing and roller fault bearing systems were determined, based on the Volterra kernel functions of $h_{1}$ and $h_{2}$, and using the second-order nuclear of $h_{2}$.

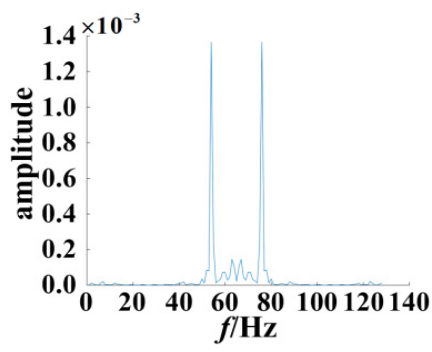

a) Normal bearing

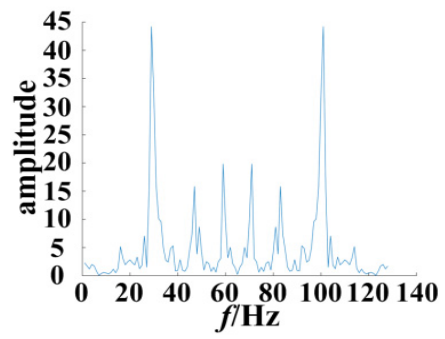

b) Damaged bearing with $0.18 \mathrm{~mm}$ diameter fault

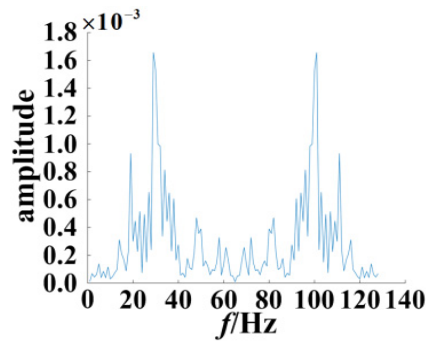

c) Damaged bearing with $0.36 \mathrm{~mm}$ diameter fault

Fig. 9. Slices of bispectrum based on the second-order kernel

The bispectrum three-dimensional map and contour map are capable of qualitatively characterizing and comparing the normal bearing and fault bearing system characteristics, however, a more quantitative description of the system characteristics is lacking. From the bispectrum three-dimensional figures, more complex display features can be observed. Thus, to more clearly observe the normal bearing and fault bearing systems, a bispectral analysis was performed using diagonal slices. The position of the selected $f=f_{1}=f_{2}$ was used to slice the bispectrum, as shown in Fig. 9. In addition to extracting useful information from the bi-spectral three-dimensional figure, computation times were effectively reduced, whilst still retaining all relevant information about the bispectrum; wherein, the normal and faulty bearings with different 
degrees of damage showed a clear contrast suggesting that failures can be successfully predicted and diagnosed.

Analysis of the bispectral slices show two peaks in the bispectral slices of the normal bearing, which appear at the frequency points $f=56 \mathrm{~Hz}$ and $f=77 \mathrm{~Hz}$. Whereas, the peak distribution of the bearing bispectral slices with $0.18 \mathrm{~mm}$ diameter damage is scattered, and there are significant peaks at $f=27 \mathrm{~Hz}, f=45 \mathrm{~Hz}, f=57 \mathrm{~Hz}, f=76 \mathrm{~Hz}, f=84 \mathrm{~Hz}$ and $f=101 \mathrm{~Hz}$. For the $0.36 \mathrm{~mm}$ damage diameter, the bispectrum slice distribution is even more dispersed, and there are significant peaks at $f=13 \mathrm{~Hz}, f=19 \mathrm{~Hz}, f=28 \mathrm{~Hz}, f=46 \mathrm{~Hz}, f=58 \mathrm{~Hz}$, $f=74 \mathrm{~Hz}, f=83 \mathrm{~Hz}, f=101 \mathrm{~Hz}$ and $f=111 \mathrm{~Hz}$. The above analysis demonstrates that the secondary phase coupling position and frequency are close to the point where the degree of damage changes, wherein the coupling phenomenon becomes more serious, and moreover there were significant differences compared to the normal bearing.

\subsection{Simulation study of noise level approximation under actual conditions}

In practical applications, the influence of the device should be taken into account. Based on the experimental results, the external noise based on different signal-to-noise ratios, $\mathrm{SNR}=12 \mathrm{~dB}$ and $\mathrm{SNR}=6 \mathrm{~dB}$, were added to the collected signals to simulate the effect of smooth operation and non-stationary operation. Using the method developed in this paper, the data was analyzed and used to produce the bispectrum based on the Volterra kernel function, as shown in Fig. 10. This was then compared to the experimental results.

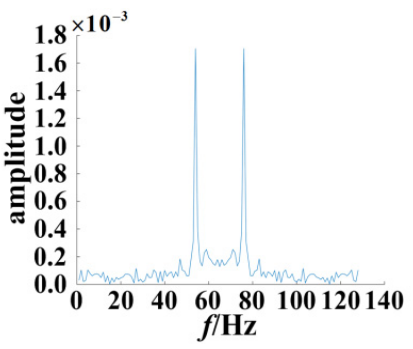

a) Normal bearing

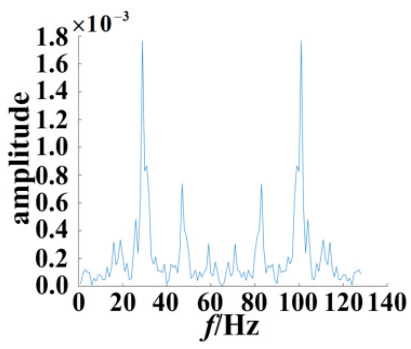

b) Damaged bearing

with $0.18 \mathrm{~mm}$ diameter fault

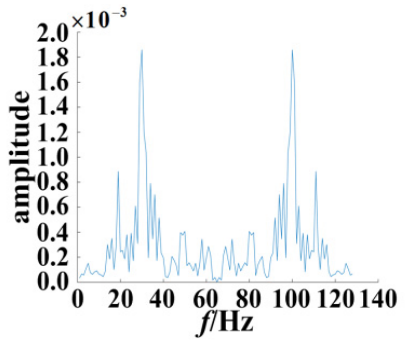

c) Damaged bearing with $0.36 \mathrm{~mm}$ diameter fault

Fig. 10. Slices of bispectrum based on the second-order kernel $(\mathrm{SNR}=12 \mathrm{~dB})$

Comparing Figs. 9 and 10, it is found that after adding the external noise with $\mathrm{SNR}=12 \mathrm{~dB}$, although some fluctuations appear, the frequency points corresponding to its peak are not affected as a whole. The bispectrum slices of the normal bearing still show the highest peaks at $f=56 \mathrm{~Hz}$ and $f=77 \mathrm{~Hz}$. Similarly, the bearing bispectral slices for the damage diameter of $0.18 \mathrm{~mm}$ have peaks at $f=28 \mathrm{~Hz}, f=46 \mathrm{~Hz}, f=57 \mathrm{~Hz}, f=75 \mathrm{~Hz}, f=83 \mathrm{~Hz}, f=101 \mathrm{~Hz}$, and other significant peaks also appear nearby. For the $0.36 \mathrm{~mm}$ diameter fault, the bispectrum slice distribution of the bearing is more dispersed, and there are significant peaks at $f=14 \mathrm{~Hz}$, $f=19 \mathrm{~Hz}, f=28 \mathrm{~Hz}, f=47 \mathrm{~Hz}, f=58 \mathrm{~Hz}, f=74 \mathrm{~Hz}, f=83 \mathrm{~Hz}, f=102 \mathrm{~Hz}$ and $f=113 \mathrm{~Hz}$. The different secondary phase coupling phenomena can be described and compared by the different degrees of damage to the fault bearing.

When external noise was present, the signal-to-noise ratio SNR $=6 \mathrm{~dB}$ was added, and the bispectrums for the different degrees of damage were produced. The peak arrangement was disorderly, and the useful information was hidden by noise; therefore, it was difficult to extract the fault features. More detailed information can be derived from the 3rd order and above for the higher order Volterra kernel function, however we do not elaborate on this here. In general, the results of the analysis, with either no external noise or adding a certain SNR based on the external noise, are consistent. The influence of external conditions can cause clutter in the signal, however the secondary coupling frequency point position does not have much impact. Under these 
conditions, the equipment has no obvious shock, and the method can ignore the influence of some of the equipment itself, and can therefore be applied to determine the faulty bearing characteristics due to different degrees of damage.

The wavelet and envelope method was used to analyze the bearing under the same experimental conditions. After adding the external noise $(\mathrm{SNR}=12 \mathrm{~dB})$, the output signal was subjected to the wavelet, denoting the envelope analysis, and compared with the proposed methods in this paper. The roller fault characteristic frequency can be defined as:

$f_{\text {inner }}=\frac{z}{2}\left(1+\frac{d}{D} \cos \alpha\right) \frac{n}{60}$.

Using the data obtained in the experiment, this was calculated as $f_{\text {inner }}=159.7 \mathrm{~Hz}$.

The wavelet base was chosen as "db6" and subjected to the Hilbert envelope analysis, as shown in Fig. 11, and compared to the multi-pulse excitation method described above.

The envelope spectrum of the normal bearing was compared to bearings with different degrees of damage, as shown in Fig. 11. The frequency point of the highest peak was at $161.2 \mathrm{~Hz}$. (Fig. 11(b-c)), which is consistent with the theoretically calculated fault frequency point. Building upon these results, the frequency point can be used to distinguish the normal bearing and roller fault bearing. A shown in Fig. 11(b) and 11(c), with the exception of the peak (161.2 Hz), the rest of the fluctuations also increased as the degree of the fault increased. However, its distribution is irregular, making it difficult to extract additional fault information. A significant advantage of the method presented in this paper is the use of Volterra series modeling together with the secondary phase coupling frequency, represented by the second order kernel function bispectrum slice figure to show the fault features. This can be used to assess the different degrees of damage to a bearing. In addition, bispectrum analysis is insensitive to noise, so it can continue to extract the characteristics of the bearing provided there is no obvious impact, and thus to a certain extent, overcome the influence of the equipment itself.

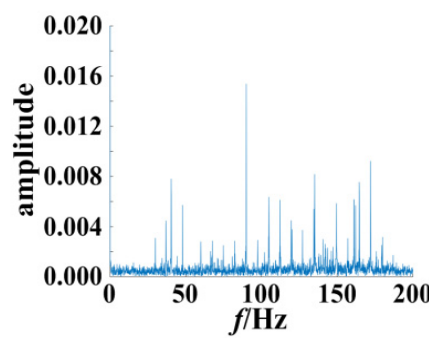

a) Normal bearing

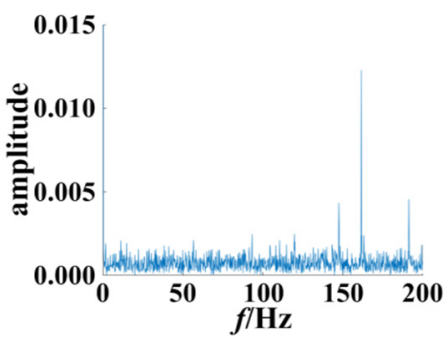

b) Bearing with $0.18 \mathrm{~mm}$ diameter fault

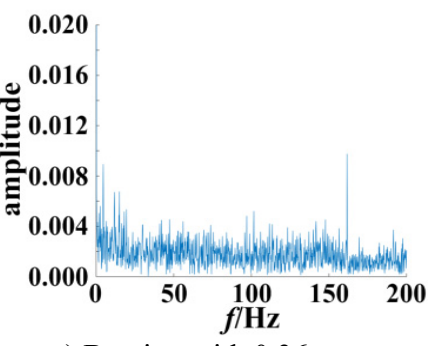

c) Bearing with $0.36 \mathrm{~mm}$ diameter fault

Fig. 11. Envelope spectrum based on different degrees of bearing damage

Haitao Wang conceived the idea. all authors discussed the results and revised the manuscript. Zhenya Kang wrote the paper. all authors discussed the results and revised the manuscript. Lichen Shi performed manuscript review. all authors discussed the results and revised the manuscript. Kun Wang provided assistance for data acquisition, data analysis and statistical analysis. all authors discussed the results and revised the manuscript. Xiao Zhang collected important background information. all authors discussed the results and revised the manuscript.

\section{Conclusions}

The Volterra series can be used to extract weak faults in nonlinear systems. Using low-order nuclei, the Volterra series was obtained using the multi-pulse excitation method, identified by the second-order and third-order nuclei of the GIRF and GFRF. The proposed method can more easily 
extract the fault characteristics of a non-linear system compared to the traditional wavelet transform method. It was found that the multi-pulse excitation method has its own unique characteristics. The method is relatively straight-forward and can simplify the filtering and selection of the wavelet for analysis. The wavelet system is very complicated, however the input and output of the system can be used to assess and extract the fault characteristics, which can then be used to determine the transfer function of the system, while neglecting any interference from the outside world.

\section{References}

[1] Aleksandrov Yu A., Kosov A. A. The stability and stabilization of non-linear, non-stationary mechanical systems. Journal of Applied Mathematics and Mechanics, Vol. 74, Issue 5, 2010, p. 553-562.

[2] Yanlin Guo, Ahsan Kareem Non-stationary frequency domain system identification using time-frequency representations. Original research article, Mechanical Systems and Signal Processing, Vol. 72, Issue 73, 2016, p. 712-726.

[3] Ruqiang Yan, Robert Gao X., Xuefeng Chen Wavelets for fault diagnosis of rotary machines: A review with applications. Signal Processing, Vol. 96, 2014, p. 1-15.

[4] Zeng Z. Z. Application of wavelet theory in roller bearing fault diagnosis. Application of Computer System, Vol. 21, Issue 7, 2012, p. 553-562.

[5] Cui B. Z., Pan H. X. Application of wavelet analysis in fault diagnosis of rolling bearing. Sci-Tech Information Development and Economy, Vol. 15, Issue 2, 2005, p. 176-178.

[6] Qiao Z. J., Pan Z. R. SVD principle analysis and fault diagnosis for bearing based on the correlation coefficient. Measurement Science and Technology, Vol. 26, 2015, p. 85014.

[7] Chen Z., Zheng S. X. The EMD signal analysis method of edge effect analysis. Journal of Data Acquisition and Processing, Vol. 18, Issue 1, 2003, p. 114-118.

[8] Saidi Lotfi, Ali Jaouher Ben, Fnaiech Farhat Bi-spectrum based-EMD applied to the non-stationary vibration signals for bearing faults diagnosis. ISA Transactions, Vol. 53, Issue 5, 2014, p. 1650-1660.

[9] Zhao Haifeng Research on Fault Feature Extraction of Non-Stationary Signal Based on NHHT. Northeast Petroleum University, 2007.

[10] He Qingbo Time-frequency manifold for nonlinear feature extraction in machinery fault diagnosis. Mechanical Systems and Signal Processing, Vol. 35, Issues 1-2, 2013, p. 200-218.

[11] Lin Jinshan, Chen Qian A novel method for feature extraction using crossover characteristics of nonlinear data and its application to fault diagnosis of rotary machinery. Mechanical Systems and Signal Processing, Vol. 48, Issues 1-2, 2014, p. 174-187.

[12] Soleimani A., Khadem S. E. Early fault detection of rotating machinery through chaotic vibration feature extraction of experimental data sets. Chaos, Solitons and Fractals, Vol. 78, 2015, p. 61-75.

[13] Li Yongbo Early fault feature extraction of rolling bearing based on ICD and tunable Q-factor wavelet transform. Mechanical Systems and Signal Processing, Vol. 86, Issue 1, 2017, p. 204-223.

[14] Shang Jun, Chen Maoyin, Ji Hongquan, Zhou Donghua, Liang Mingliang Lixihui, Xu Minqiang, Huang Wenhu Dominant trend based logistic regression for fault diagnosis in nonstationary processes. Control Engineering Practice, Vol. 66, 2017, p. 156-168.

[15] Wazwaz Abdul-Majid, Rach Randolph Two reliable methods for solving the Volterra integral equation with a weakly singular kernel. Journal of Computational and Applied Mathematics, Vol. 302, 2016, p. 71-80.

[16] Jiao L. C. Nonlinear system fault diagnosis of voltaire functional theory. Journal of Xi'an Jiaotong University, Vol. 22, Issue 3, 1988, p. 79-85.

[17] Wei R. X. On the model of nonlinear system fault diagnosis methods. Journal of Systems Engineering and Electronics, Vol. 26, Issue 11, 2004, p. 1736-1738.

[18] Al-Bugharbee Hussein, Trendafilova Irina A fault diagnosis methodology for rolling element bearings based on advanced signal pretreatment and autoregressive modelling. Journal of Sound and Vibration, Vol. 369, Issue 12, 2016, p. 246-265.

[19] Xia X. L. Mechanical equipment fault detection and diagnosis technology status and development. Coal Machinery, Vol. 28, Issue 3, 2007, p. 180-191.

[20] Shiki S. B., Jr V. L., Silva S. D. Identification of nonlinear structures using discrete-time Volterra series. Journal of the Brazilian Society of Mechanical Sciences and Engineering, Vol. 36, Issue 3, 2013, p. 523-532. 
[21] Chen S., Billings S. A., Chen S., et al. A series method to analyze nonlinear Volterra systems under periodic excitation. Proceedings of the 13th IASTED International Conference on Control and Applications, 2011.

[22] Dong Guangming, Chen Jun, Zhao Fagang A frequency-shifted bispectrum for rolling element bearing diagnosis. Journal of Sound and Vibration, Vol. 339, 2015, p. 396-418.

[23] Saidi Lotfi The deterministic bispectrum of coupled harmonic random signals and its application to rotor faults diagnosis considering noise immunity. Applied Acoustics, Vol. 122, 2017, p. 72-87.

[24] Yonghua Li, Rongqiang Jiao, Weidong Tang, Chao Cai, Jiancheng Shi, et al. Feature extraction method based on empirical mode decomposition and bispectrum analysis. Journal of Vibration, Measurement and Diagnosis, Vol. 37, 2017, p. 338-342.

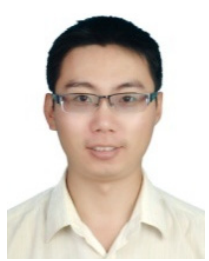

Haitao Wang received Ph.D. degree in Northwestern Polytechnical University, Xi'an, China, in 2010. Now he is a Lecturers of the College of Mechanical and Electrical Engineering at Xian University of Architecture and Technology. His current research interests include control, mechanical vibration theory and fault diagnosis.

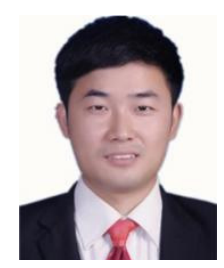

Zhenya Kang is a Postgraduate of the College of Mechanical and Electrical Engineering at Xi'an University of Architecture and Technology. His current research interests include control, Mechanical vibration theory and fault diagnosis.

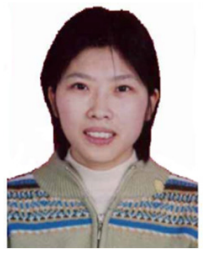

Lichen Shi is a full Professor of the College of Mechanical and Electrical Engineering at Xi'an University of Architecture and Technology. Her current research interests include control, mechanical vibration theory and fault diagnosis, vehicle dynamics and control and dynamic analysis and dynamic design of electromechanical equipment.

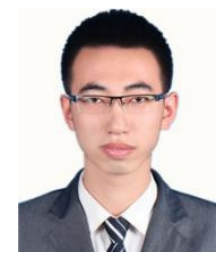

Kun Wang is a Postgraduate of the College of Mechanical and Electrical Engineering at Xi'an University of Architecture and Technology. His current research interests include control, mechanical vibration theory and fault diagnosis.

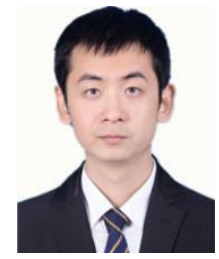

Xiao Zhang received Postgraduate degree in the College of Mechanical and Electrical Engineering at Xi' an University of Architecture and Technology. Now he works at Shanxi Automobile Group Co., Ltd. His current research interests include control, mechanical vibration theory and fault diagnosis. 